\title{
Estudios de imagen cardiaca en la pandemia COVID-19
}

\author{
Cardiac imaging studies in the COVID-19 pandemic
}

\begin{abstract}
Patricia Pérez-Soriano ${ }^{1,2 *}$, Magali Herrera-Gomar ${ }^{1,3}$, José J. Lozoya-del Rosal4, Armando I. Fajardo-Juárez ${ }^{4}$, Sergio G. Olmos-Temois ${ }^{5}$ e Isabel Carbajal-Juárez ${ }^{6}$

${ }^{1}$ Sociedad Mexicana de Cardiología; ${ }^{2}$ Cardióloga-Ecocardiografista Hospital ABC; ${ }^{3}$ Cardióloga-Ecocardiografista Hospital Médica Sur; ${ }^{4}$ Cardiología Nuclear, Centro Médico ABC; ${ }^{5}$ Cardiología e Imagen Cardiovascular no Invasiva, Centro Médico Quirúrgico de Celaya, Guanajuato; ${ }^{6}$ Departamento de Cardiología Nuclear, Instituto Nacional de Cardiología Ignacio Chávez y Servicio de Medicina Nuclear, Hospital de Especialidades, Centro Médico Nacional Siglo XXI
\end{abstract}

\begin{abstract}
Resumen
EI COVID-19 es un síndrome respiratorio agudo ocasionado por el coronavirus-2 (SARS COV2). Los diferentes métodos de imagen cardiaca han dictado recomendaciones específicas de los diferentes métodos de imagen en esta pandemia, por lo que es indispensable recalcar las recomendaciones para la realización de estos estudios.
\end{abstract}

Palabras clave: COVID-19. Ecocardiografía. Tomografía cardiaca. Cardiología nuclear. Resonancia magnética. Equipo de protección personal.

\begin{abstract}
COVID-19 is an acute respiratory syndrome caused by coronavirus-2 (SARS COV2). The different cardiac imaging methods have issued specific recommendations for the different imaging methods in this pandemic, so it is essential to emphasize the recommendations for carrying out these studies.
\end{abstract}

Key words: COVID-19. Echocardiography Cardiac tomography. Nuclear cardiology. Magnetic resonance. Personal protection equipment.

Este síndrome respiratorio agudo severo es ocasionado por el coronavirus-2 (SARS COV2) el cual es responsable de la enfermedad COVID-19 y es transmitida de persona a persona por gotas de saliva1.

Debido a que los ecocardiografistas están en un contacto muy estrecho con estos pacientes, se recomiendan medidas de protección personal indispensables para realizar los estudios. El equipo de protección personal indispensable consiste en: máscara con una eficiencia de filtración mínima equivalente a FFP2 - N95, dos pares de guantes desechables, bata impermeable de manga larga, gorro o escafandra, protección ocular ajustada de montura integral de no utilizar gafas personales, protector facial completo que permite la utilización de las gafas personales del médico. Botas para cubrir completamente el calzado ${ }^{2,3}$.

Las diferentes sociedades de ecocardiografía e imagen cardiovascular a nivel internacional han dictado ciertas recomendaciones para priorizar las indicaciones y recomendaciones de cuándo y cómo realizar un ecocardiograma durante esta pandemia protegiendo tanto
Correspondencia:

*Patricia Pérez-Soriano

E-mail: drapatriciaperezsoriano@gmail.com
Fecha de recepción: 20-04-2020

Fecha de aceptación: 29-04-2020

DOI: 10.24875/ACM.M20000059
Disponible en internet: 26-05-2020 Arch Cardiol Mex. 2020;90(Supl):41-44 www.archivoscardiologia.com 1405-9940/@ 2020 Instituto Nacional de Cardiología Ignacio Chávez. Publicado por Permanyer. Este es un artículo open access bajo la licencia CC BY-NC-ND (http://creativecommons.org/licenses/by-nc-nd/4.0/). 
al paciente como al personal de salud, resumiéndolas en lo siguiente $\mathrm{e}^{2-4}$ :

1. El estudio debe ser solicitado solo por el personal experto.

2. Debe realizarse por médicos especialistas con la finalidad de evitar repetir estudios innecesarios.

3. El estudio solo debe realizarse en caso de cambiar sustancialmente el manejo del paciente.

4. Se debe considerar el riesgo de infección y evitar el abuso en el consumo del equipo de protección personal.

5. Los estudios de imagen en pacientes no urgentes deben considerarse cancelarlos o posponerlos.

6. Se recomiendan ecocardiogramas enfocados (FOCUS) a un objetivo determinado.

7. Se recomienda un equipo pequeño, de bolsillo fácil de manejar, de limpiar y de cubrir.

8. El estudio enfocado debe incluir: evaluación de la función sistólica ventricular izquierda, evaluar alteraciones regionales de la contractilidad, dimensión telediastólica, evaluación de la fusión sistólica del ventrículo derecho (TAPSE, cambio de la fracción de acortamiento de área, dimensión telediastólica). Alteraciones valvulares por estimación visual. Derrame pericárdico.

9. Omitir el registro del monitoreo eléctrico.

10. Los ecocardiogramas transesofágicos no están contraindicados, pero deben de evitarse en lo posible.

11. Los ecocardiogramas de esfuerzo con cicloergómetro deben evitarse en lo posible por la gran diseminación de virus, debe optarse por los estudios farmacológicos.

12. Las mediciones deben realizarse offline.

13. En el reporte debe hacerse referencia que el estudio fue restringido por la enfermedad COVID-19.

14. Tener en especial cuidado en las áreas de interpretación y en el manejo de la computadora.

A medida de como avance la epidemia, se tiene seguramente que ir normando conductas de cambio en la atención de los pacientes y se tendrá que ir dando prioridad según la evaluación de primer contacto instituido en los servicios de salud, tomando en cuenta el riesgo beneficio de cada caso.

Desde su aparición en diciembre de 2019, la enfermedad causada por SARS-COV-2 representa un desafío en la atención médica por su alto requerimiento de recursos hospitalarios. Esto lleva a que se considere cuidadosamente cuándo realizar métodos diagnósticos de imagen cardiaca recomendando actualmente sólo realizar aquellos que afecten el manejo o el pronóstico del paciente.

Las recomendaciones generales son similares en todos los métodos diagnósticos: diferir estudios no urgentes, verificar el estado de salud del paciente, realizar programación vía telefónica, uso de equipo de protección personal (EPP) por el técnico y realizar limpieza adecuada del equipo utilizado.

\section{Tomografía cardiaca (TC)}

No es recomendable que estudios no urgentes como el índice de calcio coronario, el estudio anatómico de cardiopatías congénitas o la evaluación de venas pulmonares se realicen por el momento ${ }^{5,6}$.

De acuerdo a su urgencia, los estudios se clasifican en: electivos (realizar en 8 semanas o más), semiurgentes (realizar en 4 a 8 semanas) y urgentes (realizar en 4 semanas o menos). Aquellos urgentes son:

- Dolor torácico agudo y alta probabilidad de enfermedad arterial coronaria (EAC).

- Síndrome coronario crónico con alta probabilidad de eventos cardiacos mayores.

- Planeación de intervención estructural urgente.

- Evaluación de potenciales trombos intracavitarios.

- Miocardiopatía aguda, con baja a intermedia probabilidad clínica de EAC.

- Disfunción valvular protésica aguda y/o endocarditis, abscesos.

- Tumoración cardiaca maligna y planeación de biopsia o cirugía 6 .

El médico que interpreta el estudio debe evaluar los campos pulmonares para búsqueda de hallazgos sugestivos de infección por COVID-19.

\section{Cardiología nuclear}

La Sociedad Americana de Cardiología Nuclear (ASCN) junto con la Sociedad de Medicina Nuclear e Imagen Molecular (SNMMI), también han emitido recomendaciones. Si es posible, se prefiere la perfusión por PET. Se debe buscar disminuir lo más posible la estancia de los pacientes en el servicio. Hay que considerar usar protocolos de solo estrés en pacientes de bajo riesgo y corrección de atenuación para disminuir la estancia en el servicio. Se prefiere el estrés farmacológico al físico por un menor riesgo de exposición a particulas; si se requiere esfuerzo físico se debe de proteger al personal con EPP7,8.

\section{Antes de realizar el procedimiento}

- Respetar horarios de las citas y agilizar en la medida de lo posible el tiempo del estudio a llevar a cabo. 
- Sala de espera mantener (2 metros de distancia entre un paciente y otro)

- Limitar el número de acompañantes (especialmente niños y adultos mayores)

- Utilizar de preferencia batas y sábanas desechables.

- Proporcionar al paciente cubrebocas simple desde su llegada a recepción, así como sanitizar sus manos, independientemente de las condiciones clínicas en las que acuda así como el protocolo a llevar a cabo.

- Check-list

\section{MOTIVO DE SOLICITUD}

Se solicitará al enfermo o su médico tratante especifique el motivo de su estudio.

Se llevará a cabo el siguiente interrogatorio:

Es un paciente con infección confirmada por COVID-19 o con alta sospecha clínica

$\square \mathrm{SI} \square \mathrm{NO}$

Fiebre en los últimos 5 días

$\square \mathrm{SI} \square \mathrm{NO}$

Tos frecuente en los últimos 5 días

$\square \mathrm{SI} \square \mathrm{NO}$

Mialgias en los últimos 5 días

$\square \mathrm{SI} \square \mathrm{NO}$

Ha estado en contacto con portador COVID-19 en los últimos 15 a 21 días

$\square \mathrm{SI} \square \mathrm{NO}$

Ha estado en zona de riesgo de COVID-19 en los últimos 15 a 21 días

$\square \mathrm{SI} \square \mathrm{NO}$

Si alguno de los ítems es SI se considera paciente positivo o de alto riesgo: activar protocolo local de manejo clínico de paciente con SARS-CoV-2 o alto riesgo.

- Limpieza de manos del paciente con solución hidro-alcohólica

- Recordar al paciente la necesidad de contactar con el teléfono designado en su comunidad en caso de desarrollar fiebre o síntomas sugestivos de SARS-CoV-2.

\section{Medidas de protección del personal}

Sin excepción, se recomienda que todo el personal que tenga contacto con el paciente, lleve a cabo las siguientes medidas:

- Lavado de manos con solución hidro-alcohólica

- Guantes de nitrilo estándar

- Mascarilla quirúrgica (+/- gorro desechable).

\section{Si el paciente es de alto riesgo o confirmado de COVID-19}

- Lavado de manos con solución hidro-alcohólica

- Guantes de nitrilo largos o guantes estériles

- Mascarilla FFP2 (+/- gorro desechable)

- Gafas de protección

- Bata quirúrgica

\section{Al término del estudio}

Limpieza del equipo utilizado, camilla, inyector, teclado, consola, monitor etc. y los cables del ECG deben ser limpiados con detergente desinfectante con actividad viricida.

En caso de paciente confirmado como enfermo, exhaustivo de la sala.

\section{Puntos clave:}

- Reprogramar todos los estudios no urgentes

- Realizar solo estudios urgentes cuando se considera que el resultado del estudio va a cambiar el manejo inmediato del paciente

- Promover la telemedicina

- Utilizar protocolos rápidos

- Evitar prueba de estrés físico

\section{Conclusiones}

La realización de estos métodos diagnósticos de imagen cardiaca implica la potencial exposición al agente SARS-COV-2. Hay que tener en cuenta el riesgo/beneficio de la realización de los mismos. Es importante mantenerse informado sobre futuros lineamientos para protección de pacientes y personal médico.

El estudio por resonancia magnética es un método que en la actualidad es ampliamente utilizado en escenarios de patología cardiaca diversa.

Es infrecuente su uso de forma "urgente" en gran parte debido a la duración del mismo estudio. Se sugiere diferir hasta nuevo aviso aquellos estudios que no afecten de manera significativa, la evolución clínica o la conducta terapéutica del mismo. En caso de no ser posible esperar a llevar a cabo el estudio en mejores condiciones epidemiológicas, se extienden las siguientes recomendaciones.

\section{Responsabilidades éticas}

Protección de personas y animales. Los autores declaran que para esta investigación no se han realizado experimentos en seres humanos ni en animales. 
Confidencialidad de los datos. Los autores declaran que han seguido los protocolos de su centro de trabajo sobre la publicación de datos de pacientes.

Derecho a la privacidad y consentimiento informado. Los autores declaran que en este artículo no aparecen datos de pacientes.

\section{Bibliografía}

1. COVID-19 Clinical Guidance for the Cardiovascular Care Team document. https://www.acc.org// /media/Non-Clinical/Files-PDFs-Excel-MSWord-etc/2020/02/S20028-ACC-Clinical-Bulletin-Coronavirus.pdf

2. Wang $D, H u B, H u C$, et al. Clinical characteristics of 138 hospitalized patients with 2019 novel coronavirus-infected pneumonia in Wuhan, China. JAMA. Published online February 07, 2020. Doi:10.1001/jama. 2020.1585.

3. García Fernández MA, Cabrera Schulmeyer MC, Azcárate Agüero PM Documento sobre el uso de la ecocardiografía en pacientes con COVID-19. Sociedad Española de Imagen Cardiaca. Ecocardio.com. 17 marzo 2020.
4. Picard MH, Winer RB, Echocardiography in the time of COVID-19. https:// doi.org/10.1016/j.echo.2020.04.011. April 6,2020.

5. ParKer R, Lee L, Ward TJ, Lang R. Utilization and appropriateness of transthoracic echocardiography in respons to the COVID-19 pandemic. https://doi.org/10.1016/j.echo.2020.04.006. April 4, 2020

6. Choi AD, Abbara S, Branch KR, Feuchtner GM, Ghoshhajra B, Nieman K, et al. Society of Cardiovascular Computed Tomography Guidance for Use of cardiac computed tomography amidst the COVID-19 pandemic. Journal of Cardiovascular Computed Tomography, J Cardiovasc Comput. 2020 Advance online publication. https://doi.org/10.1016/j. jcct.2020.03.002.

7. Revel MP, Parkar AP, Prosch H, Silva M, Sverzelatti N, Gleeson F, et al. On behalf of the European Society of Radiology (ESR) and the European Society of Thoracic Imaging (ESTI). (2020) COVID-19 patients and the radiology department - advice from the European Society of Radiology (ESR) and the European Society of Thoracic Imaging (ESTI). European Radiology. Advance online publication. In: https://www.myesr.org/sites/default/files/2020-04/COVID\%20 and $\% 20$ Radiology $\% 20$ departments_Website $\% 20$ version $\% 20$ April\%202-2.pdf

8. Skali H, Murthy VL, Al-Mallah MH, Bateman TM, Beanlands R, Better N, et al. Guidance and best practices for nuclear cardiology laboratories during the coronavirus disease 2019 (COVID-19) pandemic: An information statement from ASNC and SMMI. https://zenodo.org/record/3738020\#.XoX62XIpBhF. Revisado el 6 de Abril, 2020.

Anexo 1. Indicaciones.

SICA reciente: evaluación de isquemia en pacientes con riesgo moderado-alto, considerados para revascularización.

Agudización de síntomas: en pacientes con clase II-IV, con riesgo pre-test intermedio), donde la angiografía es muy

Evaluación preoperatoria: cuando la cirugía es un procedimiento urgente y la revascularización es una opción viable.

Síndromes coronarios crónicos: para evaluación de isquemia, en donde el plan de revascularización no es urgente.

Reprogramar

Priorizar realización de imagen de perfusión miocárdica de acuerdo con el nivel de urgencia de cada paciente. 